\title{
Quantitative Analysis of Activated Glucocorticoid Receptors in Nasal Polyps
}

\author{
Jiyun Wang*,**, So Watanabe*, Yoshiyuki Kyo*, \\ Harumi Suzaki* \\ *Department of Otorhinolaryngology, Showa University School of Medicine, Tokyo \\ ${ }^{* *}$ Department of Otorhinolaryngology, Ningbo No.2 Hospital, China
}

\begin{abstract}
Objective : The purpose of our study was to measure the activated glucocorticoid receptor (GR) protein level in the nuclei of nasal polyp cells and compare the results in nasal polyposis patients with chronic rhinosinusitis alone and with chronic rhinosinusitis associated with asthma, before and after glucocorticoid (GC) therapy.

Methods: We used enzyme-linked immunosorbentassay (ELISA) techniques to quantitatively measure the activated-GR protein level in the nuclei of the nasal polyp cells. Nasal polyp tissues were obtained from patients with chronic rhinosinusitis alone and with both chronic rhinosinusitis and bronchial asthma. In the latter, polyp tissues were separately obtained before and after GC therapy.

Results : Our data showed no significant differences between the activated-GR protein level of the nasal polyposis patients with chronic rhinosinusitis alone and with both chronic rhinosinusitis and bronchial asthma before GC therapy. However, the activated-GR protein level was significantly upregulated after GC therapy in the patients with both chronic rhinosinusitis and bronchial asthma.

Conclusion: The result of our research clearly showed that GCs upregulated the activated-GR protein level in the nuclei of nasal polyp cells and that the upregulation is essential for the anti-inflammatory action of GCs.
\end{abstract}

Key words : Chronic rhinosinusitis, ELISA, Glucocorticoid receptor, Nasal polyps

\section{Introduction}

Chronic rhinosinusitis is characterized by inflammatory cells in the mucous membranes of the nose and paranasal sinuses and is frequently complicated by the development of nasal polyps. Although the mechanisms of the development of nasal polyps are not well understood, it has been established that allergy and inflammatory responses are implicated in polyp formation ${ }^{1-3)}$. Because of this, glucocorticoids (GCs) are frequently used in the treatment of nasal polyps, and with remarkable success : direct intra-polyp injection or aerosols dramatically shrink $50 \%$ or more of acute enlarged polyps $^{1,2)}$. Systemic GCs have also been found to be useful for the treatment of nasal polyps ${ }^{1,2)}$. The mecha- nisms underlying the efficacy of GCs in nasal polyps, however remain to be fully elucidated.

It is well known that the anti-inflammatory action of GCs is mainly mediated by glucocorticoid receptors (GRs). Before GC binding, GRs are predominantly present in the cytoplasm in the form of a large inactive complex that includes 2 molecules of hsp90. After activation by GC binding, activated GRs dissociate from hsp90 and translocate to the nucleus where they bind as a homodimer to a specific DNA sequence called the GRE (GC response element) located in the regulatory region of the target genes. The GR homodimer interacts with a basal transcription complex to enhance or inhibit the transcription of the GR target gene $e^{4)}$.

Based on the above mechanism, we now know that 
activated GRs in the nuclei are essential to anti-inflammatory action of the GC. However, there have been no reports of activated GR protein assays in nasal polyps. The purpose of this study was to measure activated GR protein levels in the cell nuclei of nasal polyps by ELISA techniques and investigate the effect of GC therapy on the results.

\section{Materials and methods}

\section{Subjects}

Nasal polyp specimens were surgically resected from 13 chronic rhinosinusitis patients (median age, 53 years ; age range, 37-69 years) who had given their written informed consent according to a study protocol approved by the Ethics Committee of Showa University. Patients who had not been treated with GCs for a minimum of one month before the surgery were enrolled in this study. Six of the patients had chronic rhinosinusitis alone (median age, 52 years ; age range, 37-69 years ; 1 female), and the other 7 were patients who had both chronic rhinosinusitis and bronchial asthma but not aspirin-induced asthma (median age, 53 years ; age range, 41-68 years ; 1 female).

In our hospital patients with severe chronic rhinosinusitis undergo endoscopic endonasal sinus surgery separately on each side at an interval of 1 week, if they wish to undergo surgery under local anesthesia. All of the subjects in this study underwent endoscopic endonasal sinus surgery under local anesthesia. The patients with chronic rhinosinusitis alone did not receive GC therapy. The patients with both chronic rhinosinusitis and bronchial asthma, who had bilaterally nasal polyps and underwent surgery twice. The set of polyps on one side was removed during the first operation, and the patients received the following postoperative GC therapy after the first operation to prevent bronchial asthma attacks and mucosal edema at the site of the surgical wound: betamethasone, $2 \mathrm{mg} /$ day, intravenous drip, for the first 3 days, and Celestamine (betamethasone $0.25 \mathrm{mg} \cdot \underline{\mathrm{d}}$-chlorpheniramine maleate $2 \mathrm{mg} /$ 1 tablet, Schering-Plough Pharmaceutical Co. Ltd., Tokyo Japan), 2 tablets/day, for the next 3-4 days. The set of polyps in the nasal cavity on the other side was removed one week later. The subjects were devided into following three groups; a group of chronic rhinosi- nusitis alone (Group 1), a group with chronic rhinosinusitis and bronchial asthma before GC therapy (Group 2), a group with chronic rhinosinusitis and bronchial asthma after GC therapy (Group 3).

Assay for activated-GR protein levels in the cell nuclei of nasal polyps.

The activated-GR protein levels in the cell nuclei of nasal polyps were measured in duplicate with commercially available ELISA test kits (Active Motif, Co. Ltd. Carlsbad, CA, USA), which contain sufficient reagents and antibodies against GR, according to the procedure recommended by the manufacturer. In brief, nasal polyps tissue was finely diced and resuspended in hypotonic buffer to swell the cell membrane and make it fragile. Detergent was added to cause leakage of the cytoplasmic proteins into the supernatant. After collection of the cytoplasmic fraction, the nuclei were lysed, and the nuclear extract was solubilized in the lysis buffer. Nuclear extract in a volume of $20 \mu \mathrm{l}(5.0 \mu \mathrm{g}$ protein) from nasal polyps was introduced into each well of 96-well microtiter plates pre-coated with an oligonucleotide that contained the GR consensus binding site (5'-GGTACAnnnTGTTCT-3'), and incubated for $1 \mathrm{~h}$ at room temperature. The GRs in nuclear extracts bind specifically to this oligonucleotide. After washing each well three times with washing buffer, $100 \mathrm{ul}$ of GR antibody was added to each well and incubated for another $1 \mathrm{~h}$ at room temperature. Anti-IgG horseradish peroxidase-conjugate in a volume of $100 \mu 1$ was then added and incubated for $1 \mathrm{~h}$ at room temperature. Finally, tetramethylbenzine solution was added, and absorbance at $450 \mathrm{~nm}$ was measured.

Statistical analysis

Data were analyzed by using Stat-View V software (Abacus, Concept, Inc, Berkeley, CA, USA). The data are shown as means \pm standard error of the mean (SEM). The statistical significance of difference between Group 1 and Group 2 was tested by the MannWhitney U test, and the difference between Group 2 and Group 3 was tested by the Wilcoxon signed-rank test. A $\underline{P}$ value less than 0.05 was regarded as statistically significant in all comparisons.

\section{Results}

1. Comparison between the activated-GR protein 
levels of the nasal polyps of the patients with chronic rhinosinusitis alone and the patients with chronic rhinosinusitis associated with bronchial asthma, before GC therapy.

There was no significant difference between the activated-GR protein levels of the nasal polyps of the patients with chronic rhinosinusitis alone and the patients with chronic rhinosinusitis associated with bronchial asthma before GC therapy (Figure 1).

2. Comparison between the activated-GR protein levels of nasal polyps before and after GC therapy in the patients with chronic rhinosinusitis associated with bronchial asthma.

The activated-GR protein levels in the nasal polyps of the chronic rhinosinusitis patients with bronchial asthma increased significantly after GC therapy $(\underline{\mathrm{P}}<0.05)$ (Figure 2).

\section{Discussion}

In this study, activated-GR was detected in the nuclei of nasal polyps before GC therapy in both the patients with chronic rhinosinusitis alone and the patients with chronic rhinosinusitis associated with bronchial asthma. This may have been because of endogenous GC secretion by the body, and this basal level of activatedGRs may be essential to maintaining human health.

The size of nasal polyps on the opposite side decrease before the second operation in the patients who received postoperative GC therapy. Consistent with this clinical effectiveness of GC, our data showed a significant increase in activated GRs in the nuclei of the nasal polyps after GC therapy in the chronic rhinosinusitis patients with bronchial asthma, and this finding is also consistent with the mechanism of GR action, that is, the increase is due to translocation of GR from the cytoplasm to the nuclei after GC binding. This study did not reveal the change in total GR level (the inactive form in the cytoplasm plus the activated form in the nuclei) after GC therapy, because the inactive GR in the cytoplasm was not measured. Another experiment will be needed to answer this question.

After GRs move into nucleus, they exert an antiinflammatory action in two ways. One way is by inhibiting the function of pro-inflammatory genes, including the genes encoding a wide variety of cytokines (IL-1,

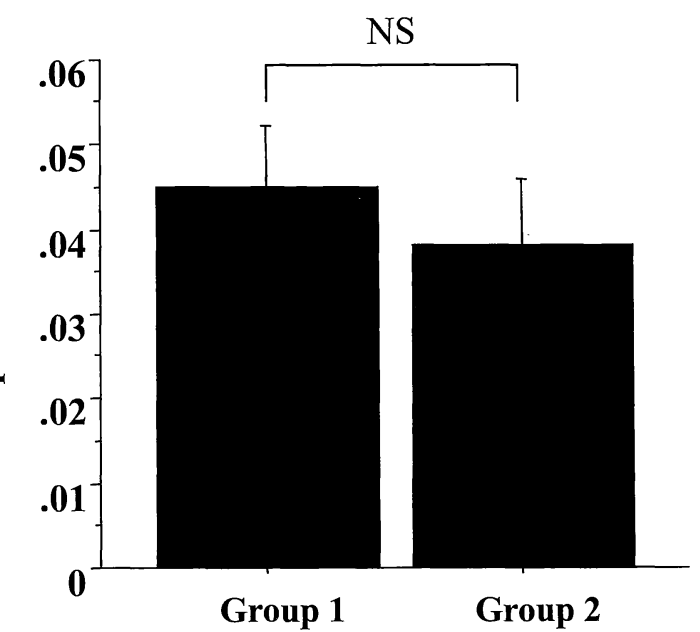

Fig. 1 Comparison between the activated-glucocorticoid receptor (GR) protein levels of nasal polyps in patients with chronic rhinosinusitis alone (Group 1) and with both chronic rhinosinusitis and bronchial asthma, before glucocorticoid therapy (Group 2). The activated-GR protein levels were assayed by ELISA. Data are shown as the means \pm SEM. The $P$ values were calculated using Mann-Whitney $U$ test. There was no significant difference between these two groups $(\underline{P}>0.05)$.
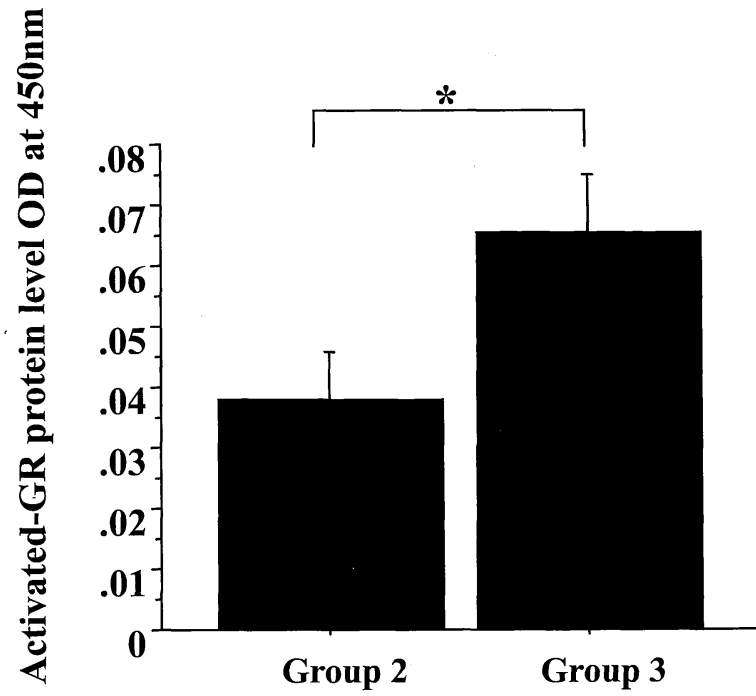

Fig. 2 Comparison of the activated-glucocorticoid receptor (GR) protein levels of nasal polyps between before (Group 2) and after the glucocorticoid therapy (Group 3) in patients with both chronic rhinosinusitis and bronchial asthma. The activated-GR protein levels were assayed by ELISA. Data are shown as the means \pm SEM. The $P$ values were calculated using Wilcoxon signedrank test. The activated GR protein levels were significantly higher in Group 3 than in Group 2 $(* \underline{\mathrm{P}}<0.05)$. 
IL-2, IL-3, IL-4, IL-5, IL-6, IL-11, IL-13, tumor necrosis factor $\alpha$, granulocyte/macrophage colonystimulating factor), chemokines (IL-8, RANTES, macrophage inflammatory protein $-1 \alpha$, monocyte chemotactic protein $[\mathrm{MCP}]-1, \mathrm{MCP}-3, \mathrm{MCP}-4$, eotaxin), adhesion molecules (intercellular adhesion molecule-1, vascular cell adhesion molecule, E-selectin), and mediatorsynthesizing enzymes (inducible nitric oxide synthase, cyclooxygenase-2, phospholipase-A2 ${ }^{5,6)}$. These genes are upregulated in nasal polyps and contribute to this pathogenesis. GCs exert their clinical efficacy by inhibiting the transcription of these genes.

The other way in which GRs exert their anti-inflammatory action is by the effect of activated GRs on antiinflammatory genes and innate immunity. The study by Mastruzzo et al. showing increased expression of TGF- $\beta$ is enlightening, because it suggests that GCs act by upregulating anti-inflammatory genes rather than just by down-regulating pro-inflammatory genes ${ }^{7)}$. Advances in DNA microarray technology have enabled further clarification of this mechanism. The first DNA microarray analysis of human tissue exposed to pharmacological doses of GCs was recently performed on nasal polyps ${ }^{8)}$, and the results showed a change in expression of some 200 genes (out of 22,000 genes analyzed) following GC administration. The gene whose expression increased the most was the gene encoding uteroglobin (also known as Clara cell protein 16, CC16). Uteroglobin is known to be secreted by Clara cells in the lungs and by nasal mucosal epithelial cells, and it has a wide-range of anti-inflammatory effects . Peptides derived from uteroglobin are among the most potent anti-inflammatory agents ${ }^{9)}$, and lower expression of uteroglobin than in healthy controls has been found not only in patients with nasal polyps but in patients with asthma as well ${ }^{10)}$.

The traditional view is that GCs suppress the body's immunity level, but recent research has shown that GCs may enhance innate immunity in airway epithelial cells in various ways ${ }^{11)}$. One mechanism of GCs' enhancing effect on human innate immunity is through the toll-like receptor (TLR) pathway. TLRs are a family of receptors involved in microbial recognition by the immune system. GCs enhance TLR expression in terms of both mRNA and protein levels, and the increased TLR is functional judging from its induction of defensins, antibiotics produced by the body that have antimicrobial activity against bacteria, fungi, and viruses ${ }^{12)}$.

In summary, GCs are effective drugs for the treatment of chronic rhinosinusitis associated with nasal polyps. GCs exert their anti-inflammatory effects in various ways that are mediated by activated GRs. Activated GRs in the cell nucleus are essential for GC action.

\section{References}

1) Lildholdt $T$, Rundcrantz H, Lindqvist $\mathrm{N}$ : Efficacy of topical corticosteroid powder for nasal polyps : a double-blind placebo-controlled study of budesonide. Clin Otolaryngol 20 : 26-30, 1995.

2 ) Bolard F, Gosset P, Lambin C, et al : Cell and cytokine profiles in nasal secretion from patients with nasal polyposis : effect of topical steroids and surgical treatment. Allergy 56: 333-338, 2001.

3 ) Jordana M, Nakano K, Nakano A, et al: Nasal polyposis : a model of chronic airway inflammation. In : Busse WW, Holgate ST, editors. Asthma and rhinitis. 2nd ed. Oxford: Blackwell Scientific Publications. 2000, 223-231.

4) Leung DY, Bloom JW : Update on glucocorticoid action and resistance. J Allergy Clin Immunol 111 : 3-22, 2003.

5 ) Pelaia G, Vatrella A, Cuda G, et al : Molecular mechanism of corticosteroid action in chronic inflammatory airway disease. Life Sci 72 : 15491561, 2003.

6 ) Benson M, Strannegard I-L, Strannegard O, et al : Topical steroid treatment of allergic rhinitis decrease nasal fluid TH2 cytokines, eosinophils, eosinophil cationic protein, and $\mathrm{IgE}$ but has no significant effect on IFN-gamma, IL-1beta, TNF-alpha, or neutrophil. J Allergy Clin Immunol 106 : 307$312,2000$.

7 ) Mastruzzo C, Greco LR, Nakano K, et al: Impact of intranasal budesonide on immune inflammatory responses and epithelial remodeling in chronic upper airway inflammation. J Allergy Clin Immunol 112 : 37-44, 2003.

8 ) Benson M, Carlsson L, Adner M, et al: Gene profiling reveals increased expression of uteroglobin and other anti-inflammatory genes in glucocorticoidtreated nasal polyps. J Allergy Clin Immunol 113 : 
1137-1143, 2004.

9 ) Miele L: New weapons against inflammation : dual inhibitors of phospholipase A 2 and transglutaminase. J Clin Invest 111: 19-21, 2003.

10) Liu Z, Kim J, Sypek J-P, et al : Gene expression profiles in human nasal polyp tissues studied by means of DNA microarray. J Allergy Clin Immunol 114: 783-790, 2004.

11) Schleimer RP: Glucocorticoids suppress inflammation but spare innate immune responses in airway epithelium. Proc Am Thorac Soc 1: 222-230, 2004.

12) Homma $T$, Kato A, Hashimoto $N$, et al: Corticosteroid and cytokines synergistically enhance toll-like receptor 2 expression in respiratory epithelial cells. Am J Respir Cell Mol Biol 31 : 463-469, 2004.

(2006年 3 月 27 日受稿, 2006年 6 月 29 日受理) Reprint and correspondence address :

Harumi Suzaki

Department of Otorhinolaryngology,

Showa University School of Medicine,

1-5-8 Hatanodai, Shinagawa-ku,

Tokyo 142-8666, Japan

Tel : +81337848676

Fax: +81337843757

E-mail : h-suzaki@med.showa-u.ac.jp (H. Suzaki) 